\title{
Small-signal converter admittance in the $p n$-frame: systematic derivation and analysis of the cross-coupling terms
}

\author{
Gabriele Amico, Agustí Egea-Àlvarez, Member, IEEE, Paul Brogan and Shuo Zhang
}

\begin{abstract}
A methodology is presented to derive the smallsignal admittance of a converter based control system in the $p n$-frame. Such a methodology makes use of a set of derived equations which link the $q d$-frame small-signal admittance data to the corresponding $p n$-frame small-signal admittance terms. Compared to existing results providing the $p n$-frame small-signal impedance of a grid connected converter, the presented technique allows a calculation of the $p n$-frame small-signal admittance (or impedance) when additional elements are present in the controller, as in many practical designs. The presented technique is therefore generic and provides a systematic way to calculate the $p n$-frame admittance regardless of the used converter control scheme. In the study, the method has been applied to assess how different parts of the controller affect the $p n$-frame admittance terms and, in particular, the cross-coupling which exists between its positive and negative sequence terms. Thereby, the results of the proposed methodology have been used to study the impact of the negative sequence current compensator on the stability performance of a grid-connected converter. Experimental tests using a real hardware set-up are used to support the obtained results.
\end{abstract}

Index Terms-positive and negative sequence impedance and admittance, voltage source converter, mirror frequency effect, dual vector current control, impedance-based stability criterion.

\section{INTRODUCTION}

$\mathbf{T}$ $\mathrm{HE}$ increase of converter-based equipment, such as solar and wind power generation or HVDC transmission systems are redefining the actual power system [1], [2]. One of the most challenging aspects of this change is the study and preservation of the grid stability. Practical issues in the stability of converter-based power systems have been reported in the literature [3]-[5] and it is likely that more cases will occur in the upcoming years (e.g. connection of power converters to weak grids [6], [7]). For this reason it is very important to have reliable and systematic tools to study, assess and prevent power converter control related interactions.

A typical configuration of a Voltage Source Converter (VSC) controller includes an inner current control loop with

G. Amico is with the Department of Electrical and Electronic Engineering, University of Strathclyde, Glasgow, UK, e-mail: gabriele.amico@strath.ac.uk

A. Egea-Àlvarez is with the Department of Electrical and Electronic Engineering, University of Strathclyde, Glasgow, UK, e-mail: agusti.egea@strath.ac.uk

P. Brogan is with Siemens Gamesa Renewable Energy, Glasgow, UK, email: Paul.Brogan@siemensgamesa.com

S. Zhang is with Pratt \& Whitney Canada, Longueuil, Canada, e-mail: zhangshuobit@gmail.com

Manuscript received Month Day, 2018; revised Month Day, 201X. the so-called dual vector current control algorithm [4]. Such a converter controller is usually implemented in the $q d$ frame and it defines a multiple-input-multiple-output (MIMO) system. The study of this system is challenging owing to the existing couplings between variables in the $q$ - and in the $d$ axis. Hence, as an effort to simplify the stability analysis, the impedance-based stability criterion has been proposed, which requires a study of the system in the $p n$-frame, see e.g. [8][10].

One pioneering work using the impedance-based method is [9], where an important assumption is that the positive and negative converter impedances/admittances are decoupled. Under the assumption that the three-phase grid is also balanced (and hence decoupled [11]), the converter-grid feedback system can be treated as two decoupled single-input-singleoutput (SISO) subsystems. A prototype of such a convertergrid interface is studied in [8] where it is demonstrated how to derive the positive and the negative sequence converter impedances. However, a generalisation of this derivation is not present to date in the literature and it is not straightforward how to extend it to the more generic case where additional components (filters or regulators) are included in the converter control loops. Moreover, there exist results highlighting non-zero cross-coupling terms between the sequence impedances/admittances, see e.g. [12]-[14]. The presence of such coupling is also discussed in [15] where it is presented in terms of a mirror frequency effect.

In view of these observations, the present paper proposes a systematic methodology to derive the converter admittance in the $p n$-frame, starting from its formulation in the $q d$-frame. This is done by application of a set of equations, whose derivation is illustrated. Comparing to existing equations (see for example [15] and [16] where the converter impedance is calculated in either the modified sequence-frame or in the $\alpha \beta$ frame, respectively), the equations proposed in this work calculate the small-signal converter impedance in the $p n$-frame. Despite the fact that these equations have been formulated in terms of admittance, they can also be used to calculate the small-signal $p n$-frame impedance terms from small-signal $q d$ frame impedance data. The equations are not dependent on the controller configuration. A practical example of their use is the derivation of the $p n$-frame small-signal converter impedance frequency response from equivalent frequency response data in the $q d$-frame, without the need to know the details of the controller architecture.

The method has been used to assess the existence of 
coupling between the positive and the negative sequence components of the converter admittance and in particular to assess how the inclusion of addtional control loops, namely the negative sequence compensator and the active power regulator, affect the Mirror Frequency Decoupled (MFD) property of the system [15], [17], [18]. Additionally, the $p n$-frame smallsignal admittance data provided by the application of the presented method have been used to study the impact of the negative sequence compensator on the system stability. Experimental results are included to validate the analysis.

The paper is organised as follows. The presented methodology and the mathematical derivation of the above-mentioned equations are presented in section II. In section III, the method is applied to a converter prototype. The results are used to study both the system MFD property for different controller formulations and the effect of the negative sequence current loop on the stability of a grid-connected converter prototype. Final conclusions are provided in section IV.

\section{DERIVATION OF SMALL-SIGNAL ADMITTANCE IN pn-FRAME}

For the purpose of illustrating the presented methodology, the converter system in Figure 1 is employed. $U_{\mathrm{I}}$ is the converter AC output voltage, $U_{\mathrm{DC}}$ is the converter DC voltage, while $U$ and $i$ are the feedback signals of the controller. The method will then be applied to the more generic case where the converter is connected to the grid.

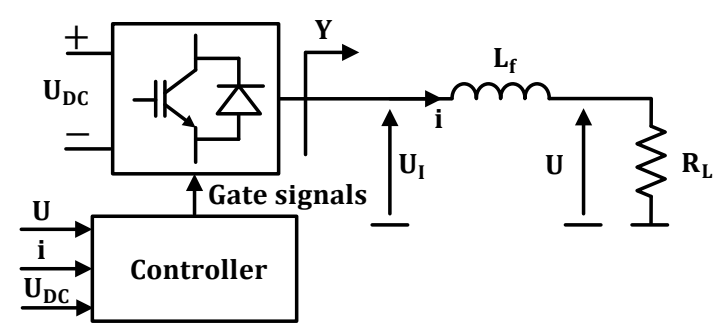

Fig. 1: One line diagram of the considered converter system.

The proposed technique makes use of a set of formulas that are based on a small-signal analytical-based model of the system in Figure 1. The small-signal admittance at the converter AC output will be considered in the following discussion. This includes not only the load resistor $R_{L}$ and the coupling reactor $L_{f}$, but also the effect of the converter feedback controller on the plant signals (in particular $U$ and i). In section II-A the definition of such admittance in the positive $q d$-frame is provided. Thereafter, in section II-B, the equations used to map the small-signal $q d$-frame admittance onto the $p n$-frame are described.

\section{A. Small-signal admittance in qd-frame}

A small-signal model of the system in Figure 1 has been built, where the dynamics have been linearised around an operating point of the system. This model has been expressed in the frequency domain and formulated in the positive $q d$ frame. The following Park transformation $T(t)$ [19] has been used to map the $a b c$-frame signals of such electrical system onto the positive $q d$-frame:

$$
T(t)=\frac{2}{3}\left[\begin{array}{lll}
\cos \left(\omega_{0} t\right) & \cos \left(\omega_{0} t-\frac{2}{3} \pi\right) & \cos \left(\omega_{0} t-\frac{4}{3} \pi\right) \\
\sin \left(\omega_{0} t\right) & \sin \left(\omega_{0} t-\frac{2}{3} \pi\right) & \sin \left(\omega_{0} t-\frac{4}{3} \pi\right)
\end{array}\right]
$$

where $\omega_{0}=2 \pi f_{0}$ is the grid frequency. The $q$-axis is aligned to phase $a$ of the measured plant voltage and leads the $d$-axis by $90^{\circ}$. In the following description, $U_{\mathrm{I}, \mathrm{qd}}, U_{\mathrm{qd}}$ and $i_{\mathrm{qd}}$ respectively denote the representations of the $a b c$-frame signals $U_{\mathrm{I}}$, $U$, and $i$, in the positive $q d$-frame. In particular, such frame corresponds to the nominal grid $q d$-frame. The signals $\Delta U_{\mathrm{I}, \mathrm{qd}}$, $\Delta U_{\mathrm{qd}}$ and $\Delta i_{\mathrm{qd}}$ identify the small-signal perturbation of $U_{\mathrm{I}, \mathrm{qd}}$, $U_{\mathrm{qd}}$ and $i_{\mathrm{qd}}$ around their steady-state values $U_{\mathrm{I}, \mathrm{qd}}^{0}, U_{\mathrm{qd}}^{0}, i_{\mathrm{qd}}^{0}$, taken at the system operation point. In the built small-signal model, a state-space representation of the electrical system equations has been applied, where an average model of the converter is used [19]. Transfer function models have been employed to describe the controller dynamics.

Such linearised model of the system has been used to calculate the small-signal $q d$-frame admittance $Y_{\mathrm{qd}}(s)$ from the $\Delta U_{\mathrm{I}, \mathrm{qd}}$ voltage to the $\Delta i_{\mathrm{qd}}$ current. It is worth noting that $Y_{\mathrm{qd}}(s)$ is dependent on the operating point of the system.

More specifically, $Y_{\mathrm{qd}}(s)$ is analytically calculated as the closed-loop transfer function matrix from $\Delta U_{\mathrm{I}, \mathrm{qd}}(s)$ to $\Delta i_{\mathrm{qd}}(s)$

$$
\Delta i_{\mathrm{qd}}(s)=Y_{\mathrm{qd}}(s) \Delta U_{\mathrm{I}, \mathrm{qd}}(s)
$$

with

$$
Y_{\mathrm{qd}}(s)=\left[\begin{array}{ll}
y_{\mathrm{qq}}(s) & y_{\mathrm{qd}}(s) \\
y_{\mathrm{dq}}(s) & y_{\mathrm{dd}}(s)
\end{array}\right]
$$

In the following section II-B, a set of formulas will be introduced to reformulate equation (2) in the $p n$-frame. These formulas provide a link between the $q d$-frame admittance terms in (3) and their expressions in the $p n$-frame.

\section{B. Translation between the qd-frame and the pn-frame}

In order to map equations (2) and (3) onto the $p n$-frame a matrix transformation is used, which consists of a combination of the $D$ and $B(t)$ matrices. Their expressions are

$$
D=\frac{1}{\sqrt{2}}\left[\begin{array}{cc}
1 & -j \\
1 & j
\end{array}\right], B(t)=\sqrt{2}\left[\begin{array}{cc}
e^{-j \omega_{0} t} & 0 \\
0 & e^{j \omega_{0} t}
\end{array}\right]
$$

The $D$ matrix maps a $q d$-frame signal on the $f b$-frame, while the $B(t)$ matrix maps a pn-frame signal on the $f b$ frame [20]. These transformations are highlighted in Figure 2 , where a diagram of the frames where electrical signals can be described is shown. It is worth mentioning that the $f b$-frame corresponds to the modified sequence-frame defined in [15]. $F$ is the Fortescue trasnformation [21], $C$ is the Clark transformation [22], $R(t)$ is the rotation matrix, which is one version of the Givens rotations [23], and is calculated as $R(t)=T(t) \cdot C^{-1}$. 


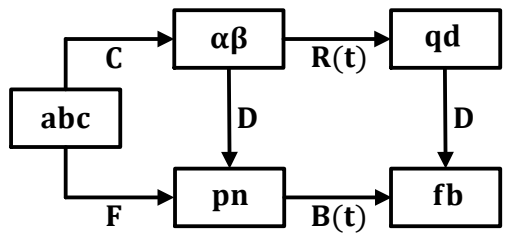

Fig. 2: Diagram of frames used to describe a three phase electrical signal analytically.

By indicating with $\Delta U_{\mathrm{I}, \mathrm{pn}}(s)$ and $\Delta i_{\mathrm{pn}}(s)$ the expressions of respectively $\Delta U_{\mathrm{I}, \mathrm{qd}}(s)$ and $\Delta i_{\mathrm{qd}}(s)$ in the $p n$-frame, based on the diagram in Figure 2, the following equations can be written

$$
\begin{aligned}
\Delta U_{\mathrm{I}, \mathrm{qd}}(s) & =\mathcal{L}\left\{D^{-1} \cdot B(t) \cdot \Delta U_{\mathrm{I}, \mathrm{pn}}(t)\right\} \\
\Delta i_{\mathrm{qd}}(s) & =\mathcal{L}\left\{D^{-1} \cdot B(t) \cdot \Delta i_{\mathrm{pn}}(t)\right\}
\end{aligned}
$$

where $\mathcal{L}$ is the Laplace transform operator [24]. Moreover, $\Delta U_{\mathrm{I}, \mathrm{pn}}(t)=\mathcal{L}^{-1}\left\{\Delta U_{\mathrm{I}, \mathrm{pn}}(s)\right\}$ and $\Delta i_{\mathrm{pn}}(t)=\mathcal{L}^{-1}\left\{\Delta i_{\mathrm{pn}}(s)\right\}$. From (2) and (5) it derives that

$$
\mathcal{L}\left\{D^{-1} \cdot B(t) \cdot \Delta i_{\mathrm{pn}}(t)\right\}=Y_{\mathrm{qd}}(s) \mathcal{L}\left\{D^{-1} \cdot B(t) \cdot \Delta U_{\mathrm{I}, \mathrm{pn}}(t)\right\}
$$

Omitting the intermediate derivation steps, which can be found in appendix A, the following set of equations are obtained

$$
\begin{aligned}
& \Delta i_{\mathrm{p}}(s)=y_{\mathrm{pp}}(s) \Delta U_{\mathrm{I}, \mathrm{p}}(s)+y_{\mathrm{pn}}(s) \Delta U_{\mathrm{I}, \mathrm{n}}\left(s-2 j \omega_{0}\right) \\
& \Delta i_{\mathrm{n}}(s)=y_{\mathrm{np}}(s) \Delta U_{\mathrm{I}, \mathrm{p}}\left(s+2 j \omega_{0}\right)+y_{\mathrm{nn}}(s) \Delta U_{\mathrm{I}, \mathrm{n}}(s)
\end{aligned}
$$

where

$$
\begin{aligned}
y_{\mathrm{pp}}(s) & =\frac{1}{2}\left\{\left[y_{\mathrm{qq}}\left(s-j \omega_{0}\right)+y_{\mathrm{dd}}\left(s-j \omega_{0}\right)\right]\right. \\
& \left.-j\left[y_{\mathrm{dq}}\left(s-j \omega_{0}\right)-y_{\mathrm{qd}}\left(s-j \omega_{0}\right)\right]\right\} \\
y_{\mathrm{pn}}(s) & =\frac{1}{2}\left\{\left[y_{\mathrm{qq}}\left(s-j \omega_{0}\right)-y_{\mathrm{dd}}\left(s-j \omega_{0}\right)\right]\right. \\
& \left.-j\left[y_{\mathrm{dq}}\left(s-j \omega_{0}\right)+y_{\mathrm{qd}}\left(s-j \omega_{0}\right)\right]\right\} \\
y_{\mathrm{np}}(s) & =\frac{1}{2}\left\{\left[y_{\mathrm{qq}}\left(s+j \omega_{0}\right)-y_{\mathrm{dd}}\left(s+j \omega_{0}\right)\right]\right. \\
& \left.+j\left[y_{\mathrm{dq}}\left(s+j \omega_{0}\right)+y_{\mathrm{qd}}\left(s+j \omega_{0}\right)\right]\right\} \\
y_{\mathrm{nn}}(s) & =\frac{1}{2}\left\{\left[y_{\mathrm{qq}}\left(s+j \omega_{0}\right)+y_{\mathrm{dd}}\left(s+j \omega_{0}\right)\right]\right. \\
& \left.+j\left[y_{\mathrm{dq}}\left(s+j \omega_{0}\right)-y_{\mathrm{qd}}\left(s+j \omega_{0}\right)\right]\right\} .
\end{aligned}
$$

It is worth mentioning that formulas (8) differ from similar equations published to date in the literature (see e.g. [15] and [16]). The equations (14) presented in [15] map a positive $q d$-frame impedance on the modified-sequence frame, and a further transformation, the $B^{-1}(t)$ transformantion in Figure 2 , is needed to go from the modified-sequence frame to the $p n$ frame. On the other hand, equations (28) and (34) in [16] link the positive $q d$-frame impedance to its $\alpha \beta$-frame counterpart. In this case, the $D$ transformation is needed to go from the $\alpha \beta$-frame to the $p n$-frame.

Equations (7-8) demonstrate that the input-output relationship between $\Delta U_{\mathrm{I}, \mathrm{pn}}(s)$ and $\Delta i_{\mathrm{pn}}(s)$ is not decoupled in the $p n$-frame. Indeed, the cross-coupling terms $y_{\mathrm{pn}}(s)$ in (7) and $y_{\mathrm{np}}(s)$ in (8) may not be zero.

There is a particular case when the cross-coupling terms $y_{\mathrm{pn}}(s)=0$ and $y_{\mathrm{np}}(s)=0$ and the $p n$-frame is decoupled. This happens if the converter is designed so that the converter controller is symmetric and $y_{\mathrm{qq}}(s)=y_{\mathrm{dd}}(s)$ and $y_{\mathrm{qd}}(s)=-y_{\mathrm{dq}}(s)$ in (2). Such a symmetric system, which is said to be Mirror Frequency Decoupled (MFD) [15], will be further studied in section III. If the $p n$-frame is decoupled, $y_{\mathrm{pp}}(s)$ and $y_{\mathrm{nn}}(s)$ in (8) will be given the name positive and negative sequence small-signal admittance.

It is emphasised that the validity of equations (7-8) does not depend on the scheme of the converter controller. As $Y_{\mathrm{qd}}(s)$ is calculated from the aforementioned small-signal model using (2-3) , equations (7-8) can always be applied to derive admittance data in the $p n$-frame. In this sense, applying equations (7-8) allows a study of comverters with complex control schemes flexibly, and generalises the results in [8], where only current-loop controller was investigated.

Both the MFD property and the effectiveness of equations (7-8) will be demonstrated in section III.

\section{ApplicATION TO A VSC SYSTEM}

In this section, equations (7-8) will be applied to demonstrate their validity and usefulness. Moreover, a laboratory test rig, where the controller is implemented in a combined DSP/FPGA system, will be used to experimentally validate the results.

In section III-A, equations (7-8) will be applied to various controller configurations to demonstrate their effectiveness without concern of details in the controller. At the same time, the MFD property of those configurations is investigated and its effect on the cross-coupling terms $y_{\mathrm{pn}}(s)$ and $y_{\mathrm{np}}(s)$ is quantified. For this purpose, in order to avoid the use of a PLL loop, which would break the MFD property of the system [15], such tests have been carried out with the converter connected to a resistive load (see Figure 1).

In section III-B, the small-signal converter admittance is employed to analyze feedback stability. The analysis aims to demonstrate how to apply the impedance-based stability criterion in [9] using the small-signal converter admittance calculated with (7-8). A comparison between the stability performances of two controller schemes, the one with, the other without the negative sequence current regulator, is presented, considering a converter connected to the grid.

\section{A. Verification of the MFD property}

A $1.5 \mathrm{~kW}$ rated converter prototype has been built, corresponding to the scheme in Figure 1. This is composed of a two level VSC, an inductive coupling filter and a resistive load. The converter controller, whose scheme is shown in Figure 3, consists of both the positive and negative sequence current control loops and of the active power regulator. The controller angle $\theta$ is generated internally by the control algorithm (i.e. no PLL loop is used). The controller scheme is based on dual current control [4]. The system and controller parameters are listed in Table I.

As described in section II, in order to calculate the small-signal $p n$-frame admittance terms defined in (8), a small-signal model of the system has been built in MATLAB. 
This model, which describes the small-signal dynamics of the system, linearised at its operating point, has been based on [19], [6] and [25]. In the model, all the signals are represented in the frequency-domain, making use of the $s$ Laplace operator [24]. Transfer functions models have been used for the controller elements, while a state-space representation of the equations of the electrical system has been employed [26]. The methodology adopted to include the negative sequence current controller in such model is outlined in appendix B. It is worth mentioning that a frame-alignment is necessary between the grid $q d$-frame and the converter $q d$-frame, as a result of the PLL dynamics. Such alignment has been included in the model, according to the theory described in [25]. The model, which is formulated in the positive $q d$-frame, is implemented as a .m MATLAB script which calculates the closed-loop transfer function matrix $Y_{\mathrm{qd}}(s)$ in (2). Hence, by applying (8), the corresponding $p n$-frame small-signal admittance terms have been obtained. Finally, by setting $s=j 2 \pi f$, the frequency responses $y_{\mathrm{pp}}(j 2 \pi f), y_{\mathrm{pn}}(j 2 \pi f), y_{\mathrm{np}}(j 2 \pi f)$ and $y_{\mathrm{nn}}(j 2 \pi f)$ have been calculated.

In order to measure such $p n$-frame frequency responses experimentally, a positive/negative sequence small-signal three-phase perturbation has been added to the $a b c$-frame PWM modulation functions of the converter. Such modulation functions represent the reference signals of the applied SVM modulation technique [27] and are calculated by the controller algorithm executed by the used DSP/FPGA control board. The frequency of the added perturbation has been varied in the $[5 \mathrm{~Hz}, 990 \mathrm{~Hz}]$ range, thus carrying out a frequency sweep test. In each iteration of the test, the small-signal perturbation has been applied for $1 \mathrm{~s}$ whilst the system is at steady-state and, simultaneously, the corresponding measured converter current $i$ has been recorded locally in the control board. As a PWM asymmetrical sampling technique has been used, with a converter switching frequency of $2.5 \mathrm{kHz}$, the $i$ data recorded during each iteration of the test is composed of 5000 samples. Thereafter, the recorded data have been exported into MATLAB to analyse their spectral composition. In particular, by having 5000 current samples for each test iteration, sampled at $5 \mathrm{kHz}$, the frequency spectrum of the collected data has been calculated with a resolution of 1 $\mathrm{Hz}$. The results of the carried out spectral analysis have allowed the calculation of the desired $p n$-frame small-signal admittance frequency responses.

TABLE I: System electrical and control parameters.

\begin{tabular}{ll}
\hline Electrical parameters & Value \\
\hline AC signal nominal frequency $f_{0}$ & $50 \mathrm{~Hz}$ \\
AC grid line voltage $U_{g}$ & $150 \mathrm{~V}_{r m s}$ \\
Grid inductance $L_{g}$ & $16 \mathrm{mH}$ \\
PWM filter resistance R & $35 \Omega$ \\
PWM filter capacitance C & $25 \mu \mathrm{F}$ \\
Coupling inductance $L_{f}$ & $2.5 \mathrm{mH}$ \\
Load resistance $R_{L}$ & $33 \Omega$ \\
Converter DC voltage $U_{D C}$ & $300 \mathrm{~V}$ \\
\hline Controller parameters & Value \\
\hline Converter switching frequency $f_{s}$ & $2.5 \mathrm{kHz}$ \\
Current loop PI proportional gain $k_{I, p}$ & $1.625 \mathrm{VA}^{-1}$ \\
Current loop PI integral gain $k_{I, i}$ & $1056.3 \mathrm{VA}^{-1} \mathrm{~s}^{-1}$ \\
Power loop PI proportional gain $k_{P, p}$ & $0.0325 \mathrm{AW}^{-1}$ \\
Power loop PI integral gain $k_{P, i}$ & $10.563 \mathrm{AW}^{-1} \mathrm{~s}^{-1}$ \\
PLL loop PI proportional gain $k_{I, p}$ & $0.129 \mathrm{radV}^{-1} \mathrm{~s}^{-1}$ \\
PLL loop PI integral gain $k_{I, i}$ & $11.6 \mathrm{radV}^{-1} \mathrm{~s}^{-2}$ \\
Low pass filter time constant $\tau_{p}$ & $0.4 \mathrm{~s}$ \\
Low pass filter time constant $\tau_{n}$ & $0.04 \mathrm{~s}^{-1}$ \\
Notch filter frequency $\omega_{N}$ & $200 \pi \mathrm{rads}^{-1}$ \\
Notch filter quality factor $Q$ & 2 \\
\hline
\end{tabular}

Different scenarios have been tested to verify the MFD property of the system, each having a different controller scheme (see Table II). In scenario 1, as only the positive sequence controller is used, the notch filters in Figure 3 have been disabled. While in scenarios 1 and 2 an operating point characterized by $\mathrm{i}_{\mathrm{q}, \text { ref }}^{+}=2 \mathrm{~A}, \mathrm{i}_{\mathrm{d} \text {,ref }}^{+}=\mathrm{i}_{\mathrm{q} \text {,ref }}^{-}=\mathrm{i}_{\mathrm{d} \text {,ref }}^{-}=0 \mathrm{~A}$ has been chosen, in scenario 3 an operating point defined with $\mathrm{P}_{\text {ref }}$ $=200 \mathrm{~W}$ has been tested, keeping $\mathrm{i}_{\mathrm{d}, \text { ref }}^{+}, \mathrm{i}_{\mathrm{q}, \text { ref }}^{-}$and $\mathrm{i}_{\mathrm{d}, \text { ref }}^{-}$equal to zero. For the three considered scenarios, Figure 4 compares
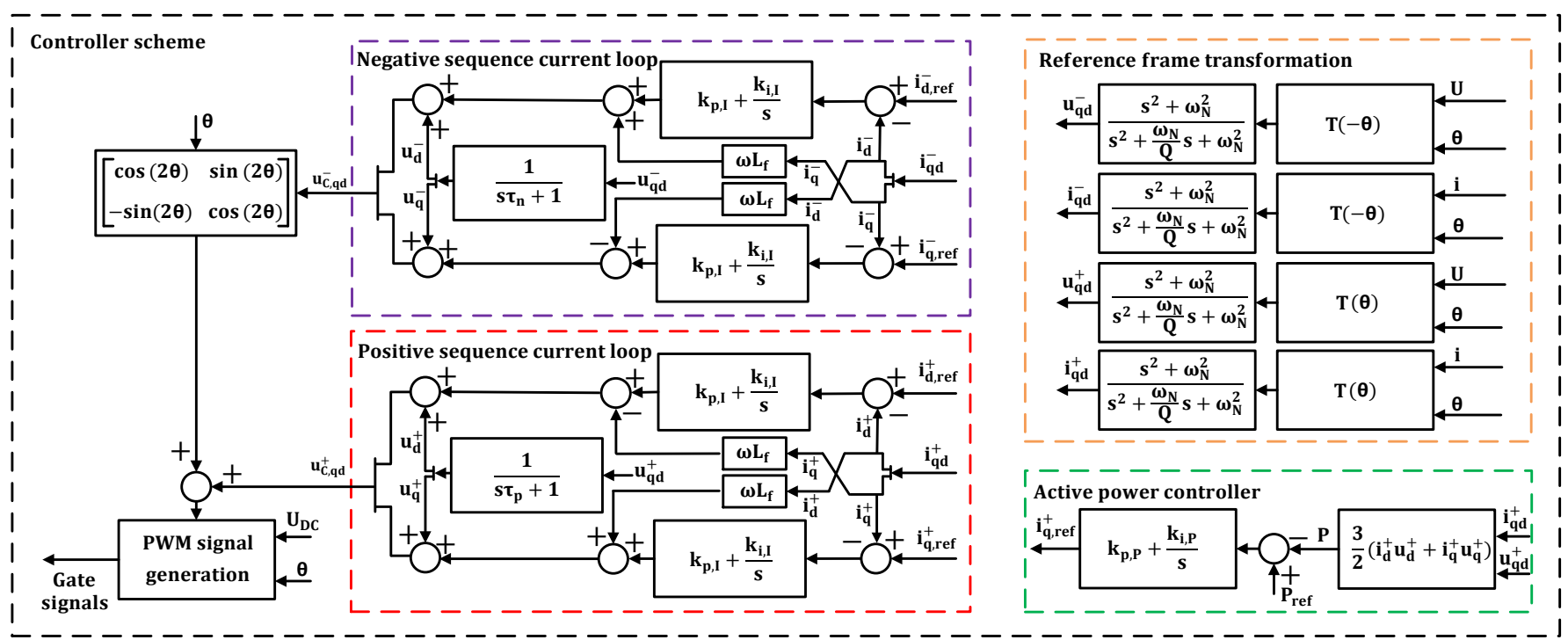

Fig. 3: Controller scheme of the converter. 

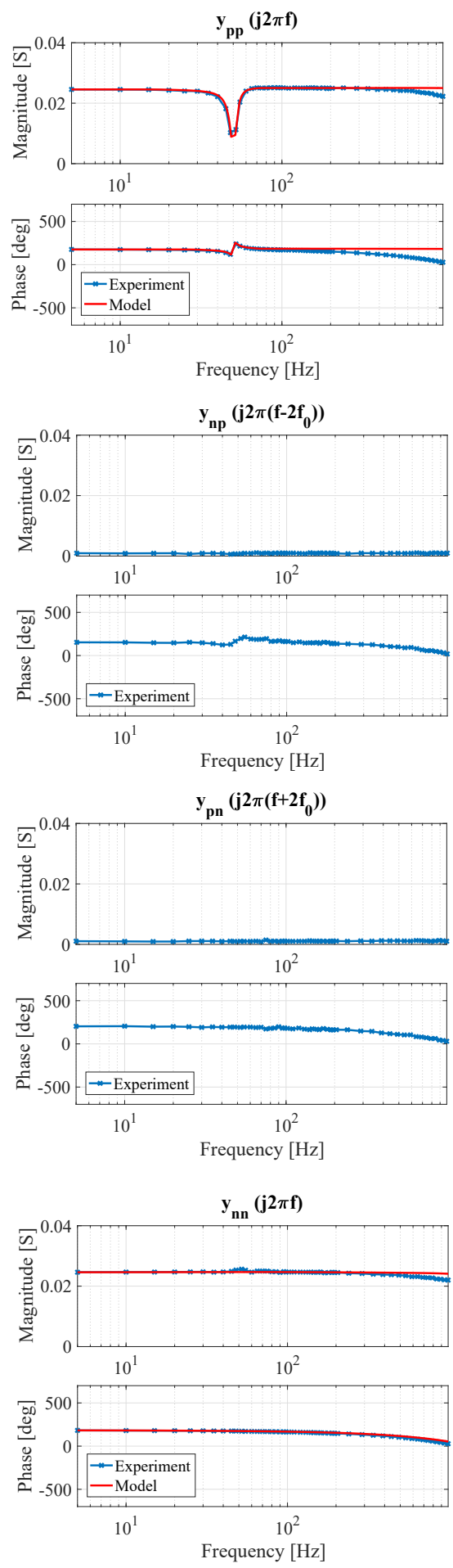

(a)
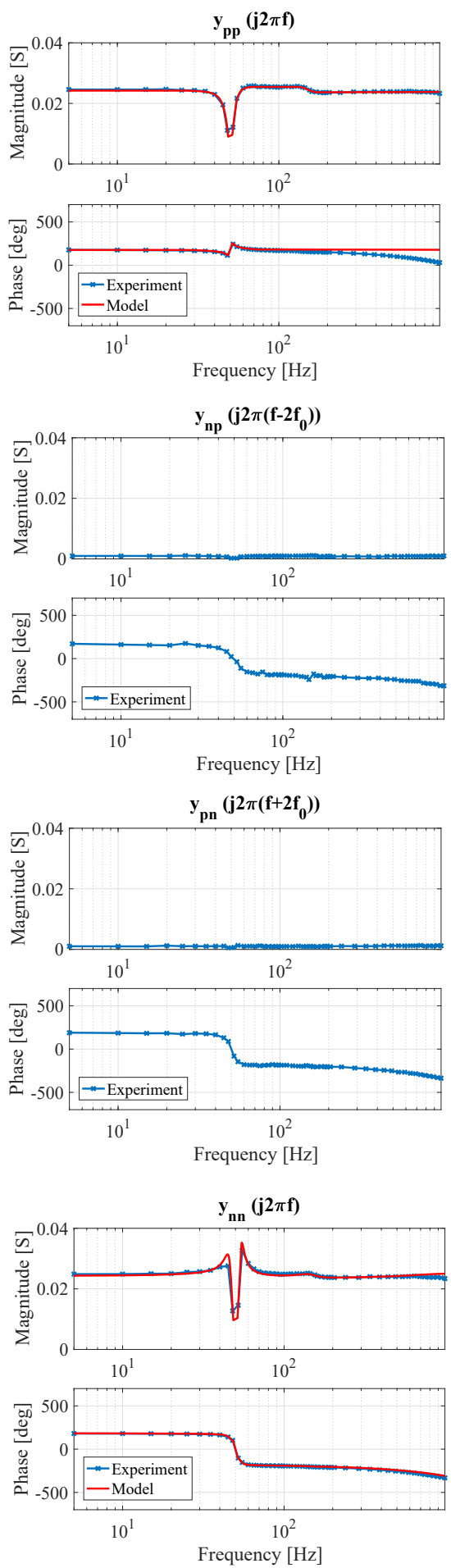

(b)
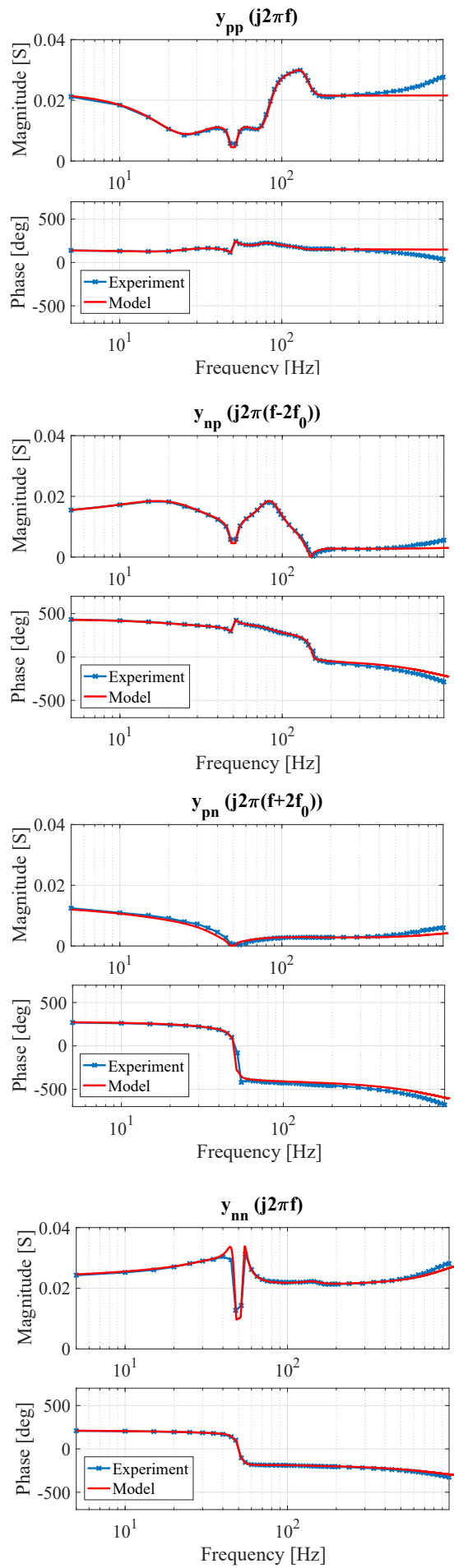

(c)

Fig. 4: Comparison between the $p n$-frame admittance frequency responses either derived experimentally or calculated analytically; (a) scenario 1, (b) scenario 2, (c) scenario 3.

the small-signal $p n$-frame admittance frequency responses calculated analytically with those derived experimentally. A substantial overlap between the two sets of results is found, which confirms both the accuracy of the applied methodology and its general applicability regardless of the used controller configuration.
In both scenarios 1 and 2 , if it is assumed that the impedances are balanced, then the system becomes MFD, as the $d$ - and $q$ - axis control loops of the used control scheme are symmetric (see Figure 3) [15]. However, nonzero coupling terms were measured, with an amplitude of about $1 \mathrm{mS}$ across the whole range of considered frequencies. 


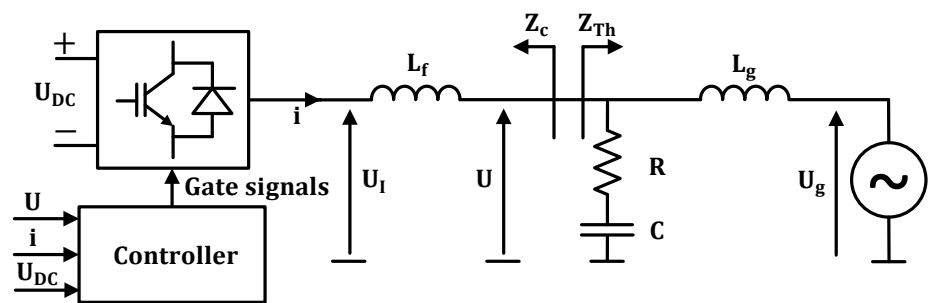

(a)

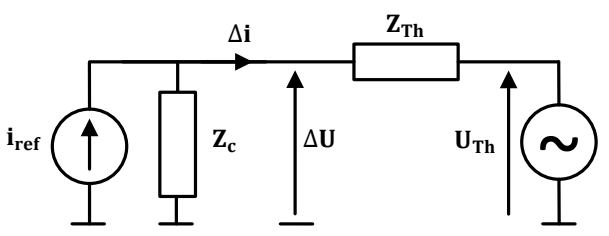

(b)

Fig. 5: (a) One line diagram of the studied grid-connected system; (b) equivalent representation of the linearised model of the electrical system, in terms of small-signal impedances.

TABLE II: Tested controller configurations

\begin{tabular}{ccc}
\hline Scenario & Neg. sequence current loop & Power loop \\
\hline 1 & Not included & Not included \\
2 & Included & Not included \\
3 & Included & Included \\
\hline
\end{tabular}

This discrepancy between the theoretical and the experimental results is attributed to a small imbalance in the electrical impedances as well as to measurement tolerances. Comparing the results from these 2 scenarios, it can be seen that the effect of the negative sequence controller is mostly noticeable in the negative sequence admittance term $y_{\mathrm{nn}}(j 2 \pi f)$, with its magnitude being strongly reduced at frequencies close to 50 $\mathrm{Hz}$ in scenario 2, while keeping a substantially constant trend in scenario 1 .

In scenario 3 , the $q d$-axis symmetry of the controller is broken by the power regulator, as this only operates on the positive $q$-axis current loop. Thereby, the system is no longer MFD, as confirmed by the larger magnitude of the coupling terms $y_{\mathrm{pn}}\left(j 2 \pi\left(f+2 f_{0}\right)\right)$ and $y_{\mathrm{np}}\left(j 2 \pi\left(f-2 f_{0}\right)\right)$. In addition to this, comparing scenario 3 with scenarios 1 and 2 , the impact of the power loop is mostly observable on the $y_{\mathrm{pp}}(j 2 \pi f)$ positive sequence term (as a result of its action being confined to the positive $q$-axis current loop).

\section{B. Stability analyis: effect of the negative sequence controller}

The effectiveness of the presented methodology to calculate the $p n$-frame admittance is now demonstrated in the stability study of a grid-connected converter system, carried out based on the impedance-based stability criterion [9]. Figure 5a shows the considered system, consisting of the converter in Figure 1 connected to a Thévenin equivalent inductive grid (whose Short Circuit Ratio is 3). An RC filter has been used to attenuate the PWM harmonics. The system parameters are listed in Table I. A PLL loop, whose block diagram is shown in Figure 6, has been used to synchronise the controller operation to the grid voltage. The controller scheme in Figure 3 has been used, with the power loop disabled. A comparison has been carried out between the controller with only the positive sequence current loop and the one with both the positive and the negative sequence current regulators. The notch filters in Figure 3 have been enabled only in the latter configuration.

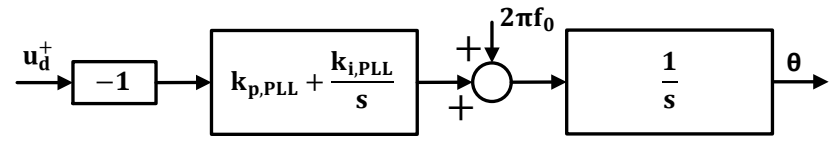

Fig. 6: Block diagram of the PLL loop.

As mentioned, the stability assessment has been carried out based on the impedance-based stability criterion [9]. Figure 5b shows the equivalent representation of the linearised model of the system in terms of small-signal impedances. The converter has been modelled in terms of its Norton equivalent, where the small-signal converter impedance $Z_{\mathrm{c}}(s)$ includes the effect of the controller on the measured voltage and current signals and it incorporates the coupling reactor connected to the AC output terminals of the converter. $Z_{\mathrm{Th}}(s)$ is the small-signal grid equivalent Thévenin impedance, which also includes the RC filter.

The open-loop gain $L(s)$ of the system has been calculated as

$$
L(s)=Y_{\mathrm{c}}(s) Z_{\mathrm{Th}}(s)
$$

where $Y_{\mathrm{c}}(s)=Z_{\mathrm{c}}(s)^{-1}$ is the small-signal converter admittance. Both $Y_{c}(s)$ and $Z_{g}(s)$ have been calculated in the $p n$-frame, applying the methodology described in section II. Accordingly, the $q d$-frame small-signal converter admittance $Y_{\mathrm{c}, \mathrm{qd}}(s)$ has been first calculated based on the $q d$-frame small-signal model of the system. Thereby, $Y_{\mathrm{c}, \mathrm{qd}}(s)$ has been mapped onto the $p n$-frame applying equations (8). For the calculation of $Z_{\mathrm{Th}}(s)$ a balanced grid impedance has been assumend. Hence, the $p n$-frame terms of $Z_{\mathrm{Th}}(s)$ are such that $z_{\mathrm{Th}, \mathrm{pp}}(s)=z_{\mathrm{Th}, \mathrm{nn}}(s), z_{\mathrm{Th}, \mathrm{pn}}(s)=z_{\mathrm{Th}, \mathrm{np}}(s)=0$, where $z_{\mathrm{Th}, \mathrm{pp}}(s)$ corresponds to the $a b c$-frame phase impedance [11]. By shifting the second equation in (7) by $-j 2 \omega_{0}$, the following matrix formulations are derived for $Y_{\mathrm{c}}(s)$ and $Z_{\mathrm{Th}}(s)$

$$
\begin{gathered}
Y_{\mathrm{c}}(s)=\left[\begin{array}{cc}
y_{\mathrm{c}, \mathrm{pp}}(s) & y_{\mathrm{c}, \mathrm{pn}}(s) \\
y_{\mathrm{c}, \mathrm{np}}\left(s-2 j \omega_{0}\right) & y_{\mathrm{c}, \mathrm{nn}}\left(s-2 j \omega_{0}\right)
\end{array}\right] \\
Z_{\mathrm{Th}}(s)=\left[\begin{array}{cc}
z_{\mathrm{Th}, \mathrm{pp}}(s) & 0 \\
0 & z_{\mathrm{Th}, \mathrm{nn}}\left(s-2 j \omega_{0}\right)
\end{array}\right]
\end{gathered}
$$

Four different operating points have been considered, as detailed in Table III.

The stability of the system has been evaluated by applying the Generalised Nyquist Criterion (GNC) to $L(s)$ [28]. It is 


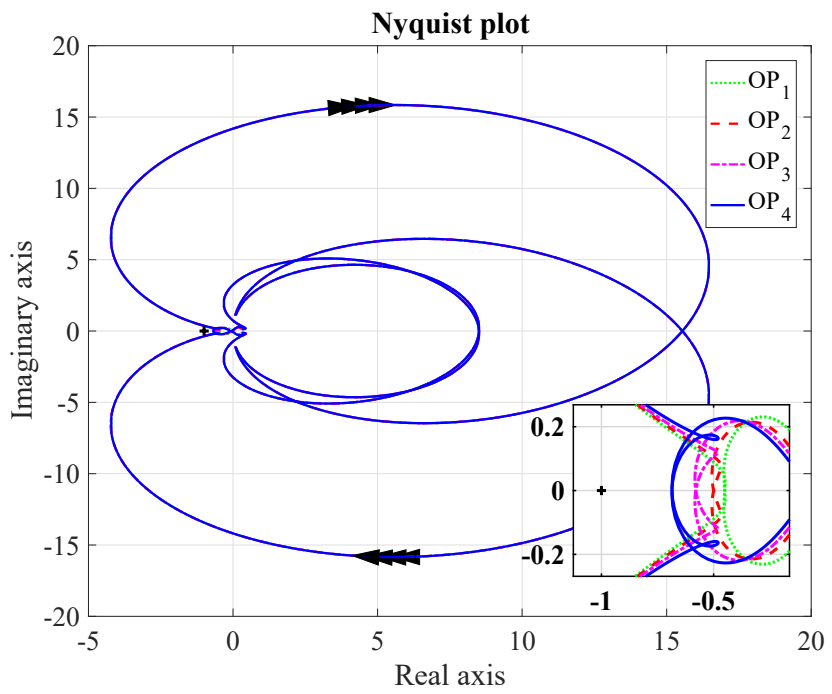

(a)

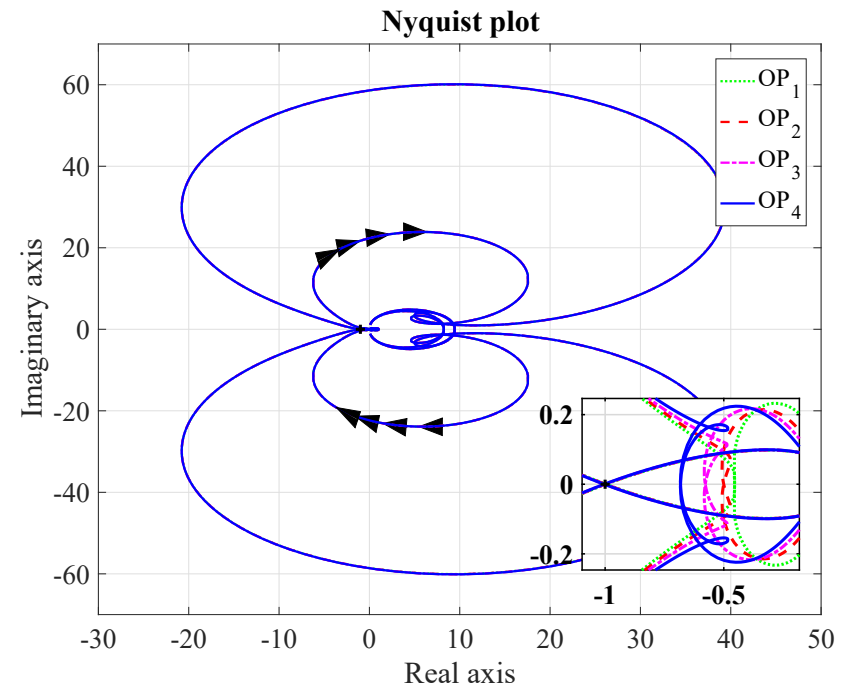

(b)

Fig. 7: Nyquist plots of the open-loop gain $L(s)$ : (a) only positive sequence, (b) both positive and negative sequence.
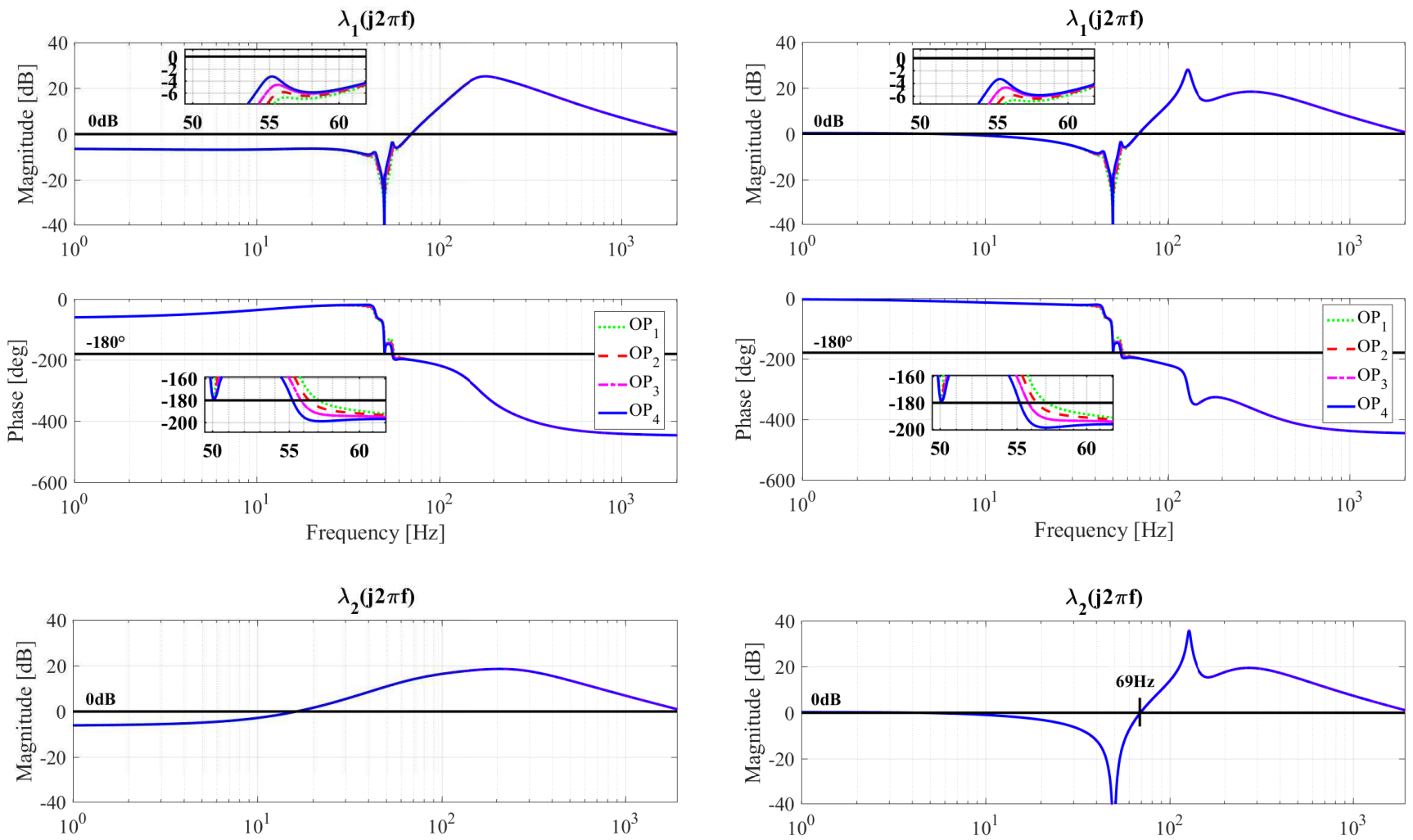

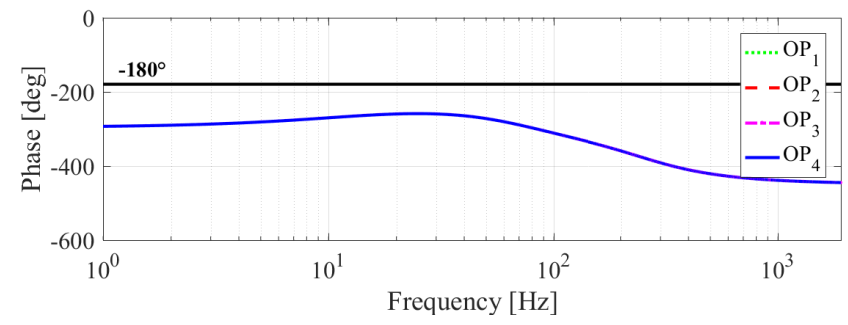

(a)

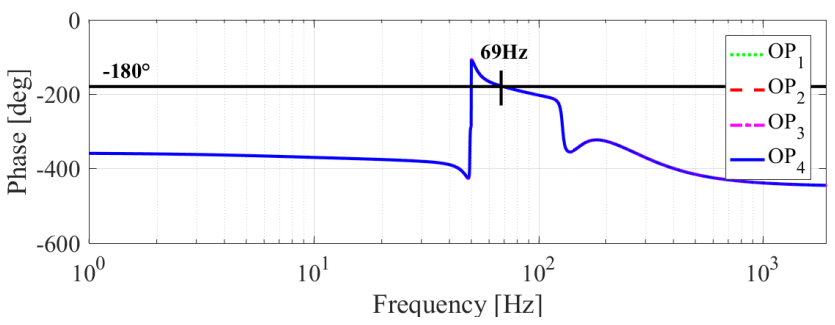

(b)

Fig. 8: Bode plots of the open-loop gain terms $\lambda_{1}(j 2 \pi f)$ and $\lambda_{2}(j 2 \pi f)$; (a) only positive sequence, (b) both positive and negative sequence. 


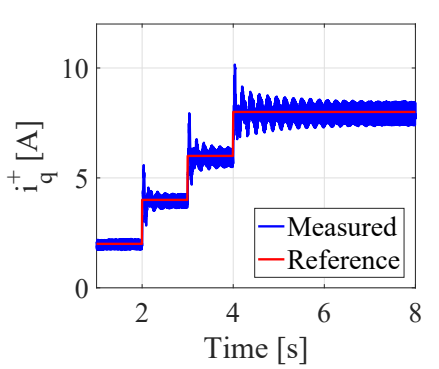

(a)

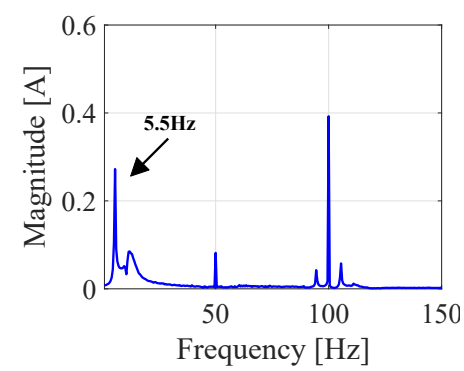

(b)

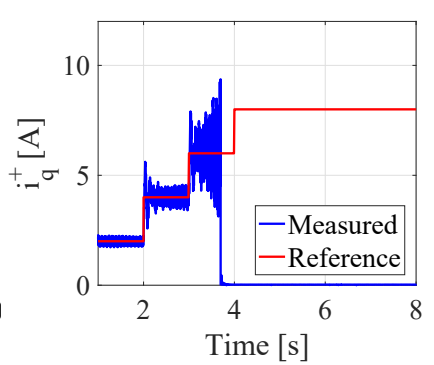

(c)

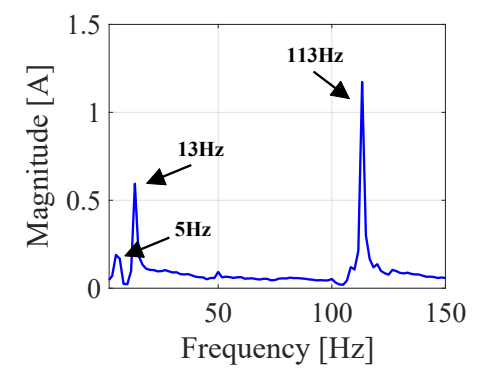

(d)

Fig. 9: Experimental results: (a) only positive sequence, (b) both positive and negative sequence.

TABLE III: Tested operating points for the system in Figure 5a

\begin{tabular}{ccc}
\hline Operating point & $\mathrm{i}_{\mathrm{q}, \text { ref }}^{+}$ & $\mathrm{i}_{\mathrm{d}, \text { ref }}^{+}, \mathrm{i}_{\mathrm{q}, \text { ref }}^{-}, \mathrm{i}_{\mathrm{d}, \text { ref }}^{-}$ \\
\hline $\mathrm{OP}_{1}$ & $2 \mathrm{~A}$ & $0 \mathrm{~A}$ \\
$\mathrm{OP}_{2}$ & $4 \mathrm{~A}$ & $0 \mathrm{~A}$ \\
$\mathrm{OP}_{3}$ & $6 \mathrm{~A}$ & $0 \mathrm{~A}$ \\
$\mathrm{OP}_{4}$ & $8 \mathrm{~A}$ & $0 \mathrm{~A}$ \\
\hline
\end{tabular}

worth highlighting that $L(s)$ is open-loop stable [29]. Figure 7 shows the Nyquist plots of $L(s)$ for the two controller configurations, i.e. the one with, the other without the negative sequence controller. While in the former case the system is on the verge of instability, in the latter case the system is robustly stable. The use of the negative sequence controller is therefore seen to reduce the stability performance of the system.

A straightforward way to investigate the critical resonance frequencies of the system is by looking at the Bode plots of the eigenvalues of $L(s)$, i.e. $\lambda_{1}(j 2 \pi f)$ and $\lambda_{2}(j 2 \pi f)$. These are shown in Figure 8 for the two considered control schemes. While $\lambda_{1}(j 2 \pi f)$ is associated to the positive sequence, $\lambda_{2}(j 2 \pi f)$ is related to the negative sequence. As it can be seen, for both of the control schemes, the critical resonance frequency of $\lambda_{1}(j 2 \pi f)$ is at $f_{p} \approx 55 \mathrm{~Hz}$, where $\angle \lambda_{1}\left(j 2 \pi f_{p}\right)$ crosses the $-180^{\circ}$ axis and $\left|\lambda_{1}\left(j 2 \pi f_{p}\right)\right|$ approaches the $0 \mathrm{~dB}$ axis as $\mathrm{i}_{\mathrm{q}, \text { ref }}^{+}$increases. On the other hand, $\lambda_{2}(j 2 \pi f)$ has a critical resonance frequency only when the negative sequence current controller is included. This critical frequency, whose value is approximately $69 \mathrm{~Hz}$, drastically reduces the stability margins of the system, making it on the verge of instability, as previously observed from the Nyquist plots in Figure $7 \mathrm{~b}$.

The analytical results have been verified experimentally. For this purpose, the laboratory converter prototype used for the tests described in section III-A has been used. Such converter has been connected to the grid according to the scheme in Figure 5a. A staircase-like increase of $\mathrm{i}_{\mathrm{q}}^{+}$,ref has been tested to reproduce the operating points in Table III. Figure 9 shows the collected experimental results together with a spectral analysis of the observed transients. Figure $9 \mathrm{a}$ and Figure $9 \mathrm{~b}$ refer to the controller with only the positive sequence controller. As it can be seen, the system is stable for all the considered operating points. The shown current spectrum magnitude, calculated for the transient starting at $\mathrm{t}=4 \mathrm{~s}$, reveals the presence of a poorly damped mode at $\approx 5.5 \mathrm{~Hz}$ in the $q d$-frame, which corresponds to a positive sequence mode at $55.5 \mathrm{~Hz}$ in the $a b c$-frame. The existence of this mode, whose damping is reduced as $\mathrm{i}_{\mathrm{q}, \text { ref }}^{+}$increases, has been correctly predicted by the presented stability study (see the Bode plot of $\lambda_{1}(j 2 \pi f)$ in Figure 8a). Figure 9c and Figure $9 d$ are associated to the control scheme where the negative sequence regulator is also included. As it can be seen, the system becomes unstable when $\mathrm{i}_{\mathrm{q}, \mathrm{ref}}^{+}=6 \mathrm{~A}$. The spectral analysis shown in Figure 9d, which is calculated for the divergent transient starting at $\mathrm{t}=3 \mathrm{~s}$, has both a spectral peak at $\approx 5 \mathrm{~Hz}$, and two further ones at $13 \mathrm{~Hz}$ and $113 \mathrm{~Hz}$. The former one is associated to a poorly damped positive sequence mode at $55 \mathrm{~Hz}$ in the $a b c$-frame, which has been correctly predicted by the stability study (see the Bode plot of $\lambda_{1}(j 2 \pi f)$ in Figure $\left.8 b\right)$. The other two modes, at 13 $\mathrm{Hz}$ and $113 \mathrm{~Hz}$, are respectively associated to a positive and a negative sequence component at $63 \mathrm{~Hz}$ in the $a b c$-frame. The highest of these peaks, i.e. the one at $113 \mathrm{~Hz}$, is the one that causes the system to be unstable, and it corresponds to the critical resonance frequency of the system, predicted to be at $69 \mathrm{~Hz}$ by the Bode plot of $\lambda_{2}(j 2 \pi f)$, shown in Figure $8 \mathrm{~b}$. The presence of the mode at $13 \mathrm{~Hz}$ is attributed to some small imbalance in the electrical impedances.

Overall, a substantial overlap is obtained between the results of the stability analysis and the experimental data, which therefore confirms the effectiveness of the proposed methodology to calculate the $p n$-frame small-signal converter admittance, necessary to apply the impedance-based stability criterion. Moreover, the experimental results have confirmed the aforementioned observation that the negative-sequence controller is seen to downgrades the stability performance of the system, despite of its benefits to mitigate unbalanced grid signals.

\section{CONCLUSION}

A systematic methodology has been presented to calculate the $p n$-frame small-signal admittance in a converter based system. This has been based on a small-signal model of the system in the positive $q d$-frame and on the application of a set of equations linking the $q d$-frame to the $p n$-frame. The illustrated method has been demonstrated to be applicable for different configurations of the converter controller. Moreover, it has been used to assess how different converter control elements (namely the negative sequence compensator and the power loop) affect the coupling between the positive and the negative sequence terms of the small-signal $p n$-frame admittance. The results provided by the method have been used to study the effect of the negative sequence current controller on the stability, showing how a reduced performance 


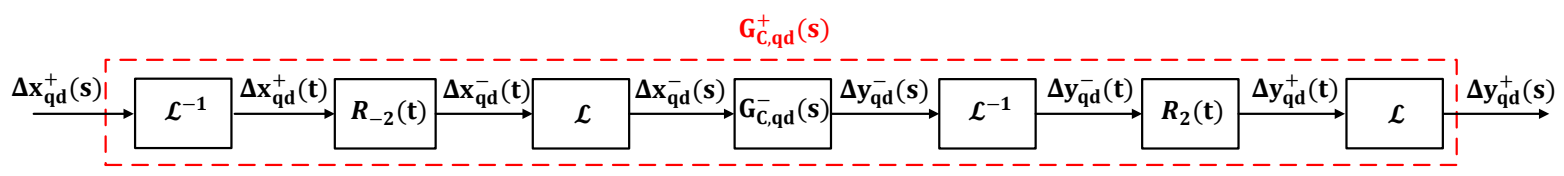

Fig. 10: Translation of the transfer function $G_{\mathrm{C}, \mathrm{qd}}^{-}$, defined in the negative $q d$-frame, onto the positive $q d$-frame.

is obtained when such compensator is applied. Experiments with a laboratory test rig have been used to confirm the effectiveness of the presented technique.

\section{APPENDIX A \\ FULL DERIVATION OF EQUATIONS (7) AND (8)}

In this section, the derivation of equations (7) and (8) reported in section II-B is presented.

Equation (2) can be formulated in the time domain as

$$
\Delta i_{\mathrm{qd}}(t)=Y_{\mathrm{qd}}(t) * \Delta U_{\mathrm{I}, \mathrm{qd}}(t)
$$

where the symbol $*$ indicates the convolution operator, $\Delta i_{\mathrm{qd}}(t)=\mathcal{L}^{-1}\left\{\Delta i_{\mathrm{qd}}(s)\right\}, \Delta U_{\mathrm{I}, \mathrm{qd}}(t)=\mathcal{L}^{-1}\left\{\Delta U_{\mathrm{I}, \mathrm{qd}}(s)\right\}$. Moreover, $Y_{\mathrm{qd}}(t)=\mathcal{L}^{-1}\left\{Y_{\mathrm{qd}}(s)\right\}$ is the impulse response of the small-signal admittance matrix $Y_{\mathrm{qd}}(s)$ [30].

Based on (5), the following relation can be derived

$$
\Delta i_{\mathrm{pn}}(t)=B^{-1}(t) D\left\{Y_{\mathrm{qd}}(t) *\left[D^{-1} B(t) \Delta U_{\mathrm{I}, \mathrm{pn}}(t)\right]\right\}
$$

which is written in the following scalar form

$$
\begin{aligned}
\Delta i_{\mathrm{p}}(t) & =\frac{1}{2} e^{j \omega_{0} t}\left\{y_{\mathrm{qq}}(t) *\left[\Delta U_{\mathrm{I}, \mathrm{p}}(t) e^{-j \omega_{0} t}+\Delta U_{\mathrm{I}, \mathrm{n}}(t) e^{j \omega_{0} t}\right]\right. \\
& \left.+y_{\mathrm{qd}}(t) *\left[j \Delta U_{\mathrm{I}, \mathrm{p}}(t) e^{-j \omega_{0} t}-j \Delta U_{\mathrm{I}, \mathrm{n}}(t) e^{j \omega_{0} t}\right]\right\} \\
& -\frac{1}{2} j e^{j \omega_{0} t}\left\{y_{\mathrm{dq}}(t) *\left[\Delta U_{\mathrm{I}, \mathrm{p}}(t) e^{-j \omega_{0} t}+\Delta U_{\mathrm{I}, \mathrm{n}}(t) e^{j \omega_{0} t}\right]\right. \\
& \left.+y_{\mathrm{dd}}(t) *\left[j \Delta U_{\mathrm{I}, \mathrm{p}}(t) e^{-j \omega_{0} t}-j \Delta U_{\mathrm{I}, \mathrm{n}}(t) e^{j \omega_{0} t}\right]\right\}, \\
\Delta i_{\mathrm{n}}(t) & =\frac{1}{2} e^{-j \omega_{0} t}\left\{y_{\mathrm{qq}}(t) *\left[\Delta U_{\mathrm{I}, \mathrm{p}}(t) e^{-j \omega_{0} t}+\Delta U_{\mathrm{I}, \mathrm{n}}(t) e^{j \omega_{0} t}\right]\right. \\
& \left.+y_{\mathrm{qd}}(t) *\left[j \Delta U_{\mathrm{I}, \mathrm{p}}(t) e^{-j \omega_{0} t}-j \Delta U_{\mathrm{I}, \mathrm{n}}(t) e^{j \omega_{0} t}\right]\right\} \\
& +\frac{1}{2} j e^{-j \omega_{0} t}\left\{y_{\mathrm{dq}}(t) *\left[\Delta U_{\mathrm{I}, \mathrm{p}}(t) e^{-j \omega_{0} t}+\Delta U_{\mathrm{I}, \mathrm{n}}(t) e^{j \omega_{0} t}\right]\right. \\
& \left.+y_{\mathrm{dd}}(t) *\left[j \Delta U_{\mathrm{I}, \mathrm{p}}(t) e^{-j \omega_{0} t}-j \Delta U_{\mathrm{I}, \mathrm{n}}(t) e^{j \omega_{0} t}\right]\right\}
\end{aligned}
$$

The Laplace transform of (14) is

$$
\begin{aligned}
\Delta i_{\mathrm{p}}(s) & =\frac{1}{2}\left\{y_{\mathrm{qq}}(s-j \omega)\left[\Delta U_{\mathrm{I}, \mathrm{p}}(s)+\Delta U_{\mathrm{I}, \mathrm{n}}(s-2 j \omega)\right]\right. \\
& \left.+y_{\mathrm{qd}}(s-j \omega)\left[j \Delta U_{\mathrm{I}, \mathrm{p}}(s)-j \Delta U_{\mathrm{I}, \mathrm{n}}(s-2 j \omega)\right]\right\} \\
& -\frac{1}{2} j\left\{y_{\mathrm{dq}}(s-j \omega)\left[\Delta U_{\mathrm{I}, \mathrm{p}}(s)+\Delta U_{\mathrm{I}, \mathrm{n}}(s-2 j \omega)\right]\right. \\
& \left.+y_{\mathrm{dd}}(s-j \omega)\left[j \Delta U_{\mathrm{I}, \mathrm{p}}(s)-j \Delta U_{\mathrm{I}, \mathrm{n}}(s-2 j \omega)\right]\right\} \\
\Delta i_{\mathrm{n}}(s) & =\frac{1}{2}\left\{y_{\mathrm{qq}}(s+j \omega)\left[\Delta U_{\mathrm{I}, \mathrm{p}}(s+2 j \omega)+\Delta U_{\mathrm{I}, \mathrm{n}}(s)\right]\right. \\
& \left.+y_{\mathrm{qd}}(s+j \omega)\left[j \Delta U_{\mathrm{I}, \mathrm{p}}(s+2 j \omega)-j \Delta U_{\mathrm{I}, \mathrm{n}}(s)\right]\right\} \\
& +\frac{1}{2} j\left\{y_{\mathrm{dq}}(s+j \omega)\left[\Delta U_{\mathrm{I}, \mathrm{p}}(s+2 j \omega)+\Delta U_{\mathrm{I}, \mathrm{n}}(s)\right]\right. \\
& \left.+y_{\mathrm{dd}}(s+j \omega)\left[j \Delta U_{\mathrm{I}, \mathrm{p}}(s+2 j \omega)-j \Delta U_{\mathrm{I}, \mathrm{n}}(s)\right]\right\}
\end{aligned}
$$

Based on the definitions given in (8), equations (15) are equal to $(7)$.

\section{APPENDIX B}

MODELling OF THE NEGATIVE SEQUENCE CONTROLLER

In this section the technique used to include the negative sequence current controller in the built MATLAB small-signal model of the system is outlined. As mentioned, such model is defined in the positive $q d$-frame, while the negative sequence controller operates on a negative $q d$-frame. This is defined by the Park transformation in (1) with $\omega_{0}$ replaced by $-\omega_{0}$ [19]. The dynamics of the negative sequence regulator have been referred to the positive $q d$-frame applying the scheme in Figure 10. By indicating with $G_{\mathrm{C}, \mathrm{qd}}^{-}(s)$ the transfer function model of a generic element of the negative sequence controller, this has the following analytical structure

$$
G_{\mathrm{C}, \mathrm{qd}}^{-}(s)=\left[\begin{array}{cc}
G_{\mathrm{C}, \mathrm{q}}(s) & 0 \\
0 & G_{\mathrm{C}, \mathrm{d}}(s)
\end{array}\right]
$$

where $G_{\mathrm{C}, \mathrm{q}}^{-}(s)$ and $G_{\mathrm{C}, \mathrm{d}}^{-}(s)$ are the transfer function models of the considered controller element in the $q$ - and $d$ - axis, respectively. Based on the symmetric structure between the $q$ - and $d$-axis current loops (see Figure 3 ), $G_{\mathrm{C}, \mathrm{q}}^{-}(s)=$ $G_{\mathrm{C}, \mathrm{d}}^{-}(s)=G_{\mathrm{C}}^{-}(s)$.

Based on the scheme in Figure 10

$$
\Delta y_{\mathrm{qd}}^{-}(s)=G_{\mathrm{C}, \mathrm{qd}}^{-}(s) \Delta x_{\mathrm{qd}}^{-}(s)
$$

where $\Delta y_{\text {qd }}^{-}(s)=\mathcal{L}\left\{\Delta y_{\text {qd }}^{-}(t)\right\}, \Delta x_{\text {qd }}^{-}(s)=\mathcal{L}\left\{\Delta x_{\text {qd }}^{-}(t)\right\}$. $\Delta x_{\mathrm{qd}}^{-}(t)$ and $\Delta y_{\mathrm{qd}}^{-}(t)$ are defined in the negative $q d$-frame, and their corresponding expressions in the positive $q d$ frame are $\Delta x_{\mathrm{qd}}^{+}(t)$ and $\Delta y_{\mathrm{qd}}^{+}(t)$, respectively. $\Delta y_{\mathrm{qd}}^{+}(s)=$ $\mathcal{L}\left\{\Delta y_{\text {qd }}^{+}(t)\right\}, \Delta x_{\text {qd }}^{+}(s)=\mathcal{L}\left\{\Delta x_{\text {qd }}^{+}(t)\right\}$.

The $\Delta y_{\mathrm{qd}}^{-}(t)$ signal can be related to $\Delta y_{\mathrm{qd}}^{+}(t)$ applying the $R_{2}(t)$ transformation [19]

$$
\Delta y_{\mathrm{qd}}^{+}(t)(t)=R_{2}(t) \Delta y_{\mathrm{qd}}^{-}(t)
$$

where

$$
R_{2}(t)=\left[\begin{array}{cc}
\cos \left(2 \omega_{0} t\right) & -\sin \left(2 \omega_{0} t\right) \\
\sin \left(2 \omega_{0} t\right) & \cos \left(2 \omega_{0} t\right)
\end{array}\right]
$$

Equivalently, the $\Delta x_{\mathrm{qd}}^{-}(t)$ signal can be related to $\Delta x_{\mathrm{qd}}^{+}(t)$ using the $R_{-2}(t)$ transformation [19]

$$
\Delta x_{\mathrm{qd}}^{-}(t)=R_{-2}(t) \Delta x_{\mathrm{qd}}^{+}(t)
$$

with $R_{-2}(t)=R_{2}^{T}(t)$.

The transfer function matrix $G_{\mathrm{C}, \mathrm{qd}}^{+}(s)$ is defined in such a way that

$$
\Delta y_{\mathrm{qd}}^{+}(s)=G_{\mathrm{C}, \mathrm{qd}}^{+}(s) \Delta x_{\mathrm{qd}}^{+}(s)
$$

Based on (17), (18) and (20), (21) can be written as

$$
\Delta y_{\mathrm{qd}}^{+}(s)=\mathcal{L}\left\{R_{2}(t) \cdot\left\{\mathcal{L}^{-1}\left\{G_{\mathrm{C}, \mathrm{qd}}^{-}(s)\right\} *\left[R_{-2}(t) \Delta x_{\mathrm{qd}}^{+}(t)\right]\right\}\right\}
$$




$$
G_{\mathrm{C}, \mathrm{qd}}^{+}(s)=\left[\begin{array}{cc}
\frac{1}{2}\left[G_{\mathrm{C}}^{-}\left(s-j 2 \omega_{0}\right)+G_{\mathrm{C}}^{-}\left(s+j 2 \omega_{0}\right)\right] & \frac{1}{2} j\left[G_{\mathrm{C}}^{-}\left(s-2 j \omega_{0}\right)-G_{\mathrm{C}}^{-}\left(s+j 2 \omega_{0}\right)\right] \\
\frac{1}{2} j\left[G_{\mathrm{C}}^{-}\left(s+j 2 \omega_{0}\right)-G_{\mathrm{C}}^{-}\left(s-j 2 \omega_{0}\right)\right] & \frac{1}{2}\left[G_{\mathrm{C}}^{-}\left(s-j 2 \omega_{0}\right)+G_{\mathrm{C}}^{-}\left(s+j 2 \omega_{0}\right)\right]
\end{array}\right]
$$

Omitting the intermediate derivation steps, the final expression of $G_{\mathrm{C}, \mathrm{qd}}^{+}(s)$ in (21) is given in (23). The illustrated method has been applied to project the transfer function model of each element of the negative sequence current controller onto the positive $q d$-frame.

\section{REFERENCES}

[1] P. S. Georgilakis, "Technical challenges associated with the integration of wind power into power systems," Renewable and Sustainable Energy Reviews, vol. 12, no. 3, pp. 852-863, 2008.

[2] J. Zhu and C. Booth, "Future multi-terminal hvdc transmission systems using voltage source converters," in Universities Power Engineering Conference (UPEC), 2010 45th International. IEEE, 2010, pp. 1-6.

[3] C. Buchhagen, C. Rauscher, A. Menze, and J. Jung, "Borwin1 - first experiences with harmonic interactions in converter dominated grids," in International ETG Congress 2015; Die Energiewende - Blueprints for the new energy age, 2015.

[4] P. Brogan, "The stability of multiple, high power, active front end voltage sourced converters when connected to wind farm collector systems," in Proc. EPE Wind Energy Chapter Seminar, 2010.

[5] E. Mollerstedt and B. Bernhardsson, "Out of control because of harmonics-an analysis of the harmonic response of an inverter locomotive," IEEE Control Systems, vol. 20, no. 4, pp. 70-81, 2000.

[6] A. Egea-Alvarez, S. Fekriasl, F. Hassan, and O. Gomis-Bellmunt, "Advanced vector control for voltage source converters connected to weak grids," IEEE Transactions on Power Systems, vol. 30, no. 6, pp. 3072-3081, 2015.

[7] L. Zhang, L. Harnefors, and H.-P. Nee, "Power-synchronization control of grid-connected voltage-source converters," IEEE Transactions on Power systems, vol. 25, no. 2, pp. 809-820, 2010.

[8] M. Cespedes and J. Sun, "Impedance modeling and analysis of gridconnected voltage-source converters," IEEE Transactions on Power Electronics, vol. 29, no. 3, pp. 1254-1261, March 2014.

[9] J. Sun, "Impedance-based stability criterion for grid-connected inverters," IEEE Transactions on Power Electronics, vol. 26, no. 11, pp. 30753078, 2011.

[10] M. Cespedes and J. Sun, "Renewable energy systems instability involving grid-parallel inverters," in Applied Power Electronics Conference and Exposition, 2009. APEC 2009. Twenty-Fourth Annual IEEE. IEEE, 2009, pp. 1971-1977.

[11] J. L. Kirtley, Electric power principles: sources, conversion, distribution and use. John Wiley \& Sons, 2011.

[12] M. K. Bakhshizadeh, X. Wang, F. Blaabjerg, J. Hjerrild, L. Kocewiak, C. L. Bak, and B. Hesselbaek, "Coupling in phase domain impedance modeling of grid-connected converters," Transactions on Power Electronics, vol. 31, no. 10, pp. 6792-6796, 2016.

[13] X. Wang, L. Harnefors, F. Blaabjerg, and P. C. Loh, "A unified impedance model of voltage-source converters with phase-locked loop effect," in Energy Conversion Congress and Exposition (ECCE), 2016 IEEE. IEEE, 2016, pp. 1-8.

[14] F. B. Xiongfei Wang, Lennart Harnefors, "Unified impedance model of grid-connected voltage-source converters," Transactions on Power Electronics, vol. 33, no. 2, pp. 1775-1787, 2018.

[15] A. Rygg, M. Molinas, C. Zhang, and X. Cai, "A modified sequencedomain impedance definition and its equivalence to the dq-domain impedance definition for the stability analysis of ac power electronic systems," Journal of Emerging and Selected Topics In Power Electronics, vol. 4, no. 4, pp. 1383-1396, 2016.

[16] X. Wang, L. Harnefors, and F. Blaabjerg, "Unified impedance model of grid-connected voltage-source converters," IEEE Transactions on Power Electronics, vol. 33, no. 2, pp. 1775-1787, 2018.

[17] C. Zhang, X. Cai, A. Rygg, and M. Molinas, "Sequence domain siso equivalent models of a grid-tied voltage source converter system for small-signal stability analysis," IEEE Transactions on Energy Conversion, vol. 33, no. 2, pp. 741-749, 2018.

[18] A. Rygg, M. Molinas, C. Zhang, and X. Cai, "On the equivalence and impact on stability of impedance modeling of power electronic converters in different domains," IEEE Journal of Emerging and Selected Topics in Power Electronics, vol. 5, no. 4, pp. 1444-1454, 2017.
[19] A. Yazdani and R. Iravani, Voltage-sourced converters in power systems: modeling, control, and applications. John Wiley \& Sons, 2010.

[20] G. C. Paap, "Symmetrical components in the time domain and their application to power network calculations," IEEE Transactions on power systems, vol. 15, no. 2, pp. 522-528, 2000.

[21] W. V. Lyon, Applications of the method of symmetrical components. McGraw-Hill book company, inc., 1937.

[22] W. Duesterhoeft, M. W. Schulz, and E. Clarke, "Determination of instantaneous currents and voltages by means of alpha, beta, and zero components," Transactions of the American Institute of Electrical Engineers, vol. 70, no. 2, pp. 1248-1255, 1951.

[23] C. F. Van Loan, "Matrix computations (johns hopkins studies in mathematical sciences)," 1996.

[24] W. Hurewicz and H. Wallman, Dimension theory. Princeton university press Princeton, 1948, vol. 1969.

[25] L. Zhang, "Modeling and control of vsc-hvdc links connected to weak ac systems," Ph.D. dissertation, KTH, 2010.

[26] W. Jacqueline, A. Johnson Michael, and K. Reza, "Control engineering: An introductory course," 2002.

[27] D. G. Holmes and T. A. Lipo, Pulse width modulation for power converters: principles and practice. John Wiley \& Sons, 2003, vol. 18.

[28] S. Skogestad and I. Postlethwaite, Multivariable feedback control: analysis and design. Wiley New York, 2007, vol. 2.

[29] B. Wen, D. Boroyevich, R. Burgos, P. Mattavelli, and Z. Shen, "Inverse nyquist stability criterion for grid-tied inverters," IEEE Transactions on Power Electronics, vol. 32, no. 2, pp. 1548-1556, 2017.

[30] R. N. Bracewell and R. N. Bracewell, The Fourier transform and its applications. McGraw-Hill New York, 1986, vol. 31999.

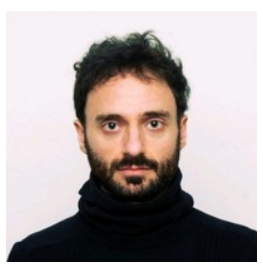

Gabriele Amico received the degree in Electronic Engineering from Polytechnic University of Milan in Italy in 2006. He worked for the Oil and Gas industry from 2006 until 2014 and received his MSc in Wind Energy Systems from University of Strathclyde in Glasgow (UK) in 2015. He is currently pursuing his $\mathrm{PhD}$ degree with the EPSRC Centre for Doctoral Training in Wind \& Marine Energy Systems and with funding provided by Siemens Gamesa Renewable Energy.

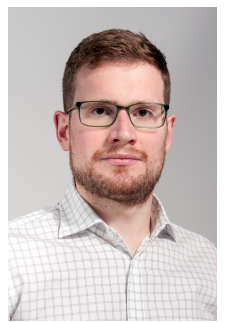

Agustí Egea-Àlvarez is Strathclyde Chancellors fellow (Lecturer) at the electronic \& electrical engineering department and member of the PEDEC group. He obtained his $\mathrm{BSc}$, MSc and $\mathrm{PhD}$ from the Technical University of Catalonia in Barcelona in 2008, 2010 and 2014 respectively. In 2015 he was a Marie Curie fellow in the China Electric Power Research Institute (CEPRI) in Beijing. Before he joined Strathclyde, he was a converter control engineer with Siemens Gamesa Renewable Energy.

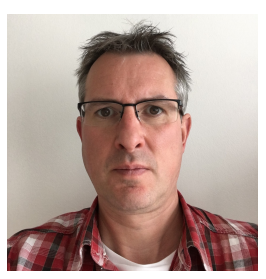

Paul Brogan received Meng from Glasgow University, Scotland in 1993, and $\mathrm{PhD}$ from University College Cork, Ireland in 1998. Since then he has been working on low voltage power converters and their control and is presently working for Siemens Gamesa Renewables Limited. 
Shuo Zhang received the B.S. and M.S. degree from Beijing Institute of Technology, Beijing, China in control theory \& engineering and the Ph.D. degree in control theory from University of Groningen, the Netherlands, in 2005, 2007, and 2013, respectively. From 2013 to 2017 he was a development engineer in power and control system in Siemens Wind Power (now called Siemens Gamesa Renewable Energy), Glasgow (UK). He is mainly interested in modelling and feedback synthesis of dynamical systems in various industrial sectors. 